Bull. Mater. Sci., Vol. 19, No. 6, December 1996, pp. 1047-1058. (C Printed in India.

\title{
Microclusters as superatoms for developing new materials
}

\author{
VIJAY KUMAR \\ Materials Science Division, Indira Gandhi Centre for Atomic Research, Kalpakkam 603 102, \\ India
}

\begin{abstract}
With the discovery of solid $\mathrm{C}_{60}$, efforts are being made to develop new clusters and molecules which could be assembled to form new materials. Here we present some recent developments in this direction and discuss bonding in such materials.
\end{abstract}

Keywords. Magic clusters; superatoms; fullerenes; cluster materials; electronic structure; cluster abundance.

\section{Introduction}

Over the centuries man has discovered 110 elements which constitute materials we see around us, but the quest for finding new ones is never ending. Attempts to synthesize elements beyond the heaviest naturally-occurring uranium have resulted in 18 manmade elements (see Seaborg 1963; and articles in Morss and Fuger 1992) and there are predictions of the existence of many more super heavy elements. However, most of these elements have a short life time, and, therefore except for a few, such as plutonium, their practical utility is very limited. On the other hand, material scientists have used various techniques of mixing different elements such as rapid solidification, mechanical mixing, ion beam mixing, layer deposition etc. besides the conventional ones, to prepare new materials with sometimes very surprising properties such as the finding of the solids with non-crystallographic long range orientational order by Shechtman et al (1984) and the exciting discovery of high temperature superconductors (Bednorz and Muller 1986; Wu et al 1987). More recently research on clusters has led to the finding of a new phase of carbon, the solid $\mathrm{C}_{60}$ (Krätschmer et al 1990), which is semiconducting and becomes a superconductor (Hebard et al 1991; Rosseinsky et al 1991) when doped with alkali metals at relatively high temperatures $\left(T_{\mathrm{c}} \approx 30 \mathrm{~K}\right)$ and an insulator on further doping (Zhou et al 1991). This has opened a new direction in the development of novel materials in which instead of atoms, microclusters (here we consider clusters with $\approx 10$-100 atoms) or molecules could be used as the building blocks, which we refer to as superatoms. As compared to atoms, the possibilities of preparing such superatoms are enormous as these could exist with various combinations of atoms and sizes.

Subsequent to the development of solid $\mathrm{C}_{60}$, single crystals of $\mathrm{C}_{70}$ have also been produced by Jiang et al (1995) even though the intensity of the $\mathrm{C}_{70}$ molecule in a mass spectrum (see subsequent sections) is much less as compared to $\mathrm{C}_{60}$. Efforts are now being made (Guo et al 1992a, b; Khanna and Jena 1992; Wei et al 1992; Gong and Kumar 1993) to develop other clusters which could potentially be used to form new materials. These developments have raised several interesting questions that one would like to answer. Some of these are: Are there criteria to find new superatoms? How will such superatoms interact with each other and with other elements? How the properties of solids derived from such superatoms would differ from conventional solids due to the structure of superatoms and so on? Also there are a large number of cluster compounds already known (Simon 1981), and some complex materials have clusters in abundance 
(Pearson 1973; Gong and Kumar 1994). Therefore, research on these problems can bring a new perspective in the understanding of a variety of materials and may lead to the development of new ones.

From the point of view of the criteria to find superatoms, studies on various elemental and binary clusters have shown (articles in Jena et al 1992; Kumar et al 1993) that their mass spectra generally exhibit strong abundance of some clusters such that clusters with one more atom are much less abundant. Such clusters have been referred to as 'magic' clusters (Echt et al 1981). For metals, the jellium model (de Heer et al 1987) has given a simple explanation for the magic clusters in terms of the completion of electronic shells, very similar to the nuclear shell model (Burcham 1973) and it can be used quite successfully for understanding the stability and abundance of $s-p$ bonded metal clusters. However, for semiconductors such an understanding is yet to be developed as the bonding character itself changes with the size of clusters. Clusters of ionic solids tend to have the same structure as in the bulk, whereas those of metals and semiconductors, often, have much different structures and electronic properties. Therefore, these are interesting from the point of view of developing new materials. Here we focus on clusters of some metals and fullerenes and first discuss the magic clusters in a jellium model. Results of studies where an effort has been made for making such superatoms and their solids are then discussed. A few results are presented on the interactions between superatoms which suggest van der Waals, covalent as well as ionic bonding between clusters.

\section{The magic clusters}

As in the case of nuclei, some atomic clusters exhibit large abundance. Knight et al (1984) found $\mathrm{Na}$ clusters with $8,20,40,58, \ldots$ atoms to be magic (figure 1a). This can be understood in terms of a jellium model in which the ionic charges are smeared into a uniform spherical positive background. The solution of the one electron equations of motion in this spherical potential leads to the electronic eigenvalues in the order, $1 s, 1 p, 1 d, 2 s, 1 f, 2 p, 1 g, \ldots$ These levels are successively filled with $2,8,18,20,34$, $40,58, \ldots$ electrons. Since an alkali atom has one valence electron, the abundance of $\mathrm{Na}$ clusters with 8,20 and 40 atoms has been explained to be due to the completion of the $1 p, 2 s$ and $2 p$ shells (figure $1 \mathrm{~b}$ ). If the gap between the highest occupied and the lowest unoccupied molecular orbitals (HOMO-LUMO) is large, then the clusters are more stable and therefore more abundant. For clusters of materials such as $\mathrm{Si}$ and $\mathrm{C}$, the jellium model is not suitable. Atomistic calculations have been performed to study their structure and stability (see Kumar 1994). We present here results on some magic clusters which have been or could be assembled into solids.

\subsection{Icosahedral $\mathrm{Al}_{13}$ clusters}

For developing solids, one would like to have very stable clusters with high symmetry (Kumar 1995). An interesting example is $\mathrm{Al}_{13}$, which is a nearly regular icosahedron(Yi et al 1991; Prasad et al 1995). It has 39 valence electrons which is one short of the shell closing in the jellium model. $\mathrm{Al}_{13}^{-}$is found to be strongly abundant in the mass spectrum (Nakajima et al 1991a) shown in figure 2. Also $\mathrm{Al}_{12} \mathrm{~B}^{-}$has been found to be abundant in boron doped aluminium clusters (Nakajima et al 1991b). Khanna and Jena (1992) 


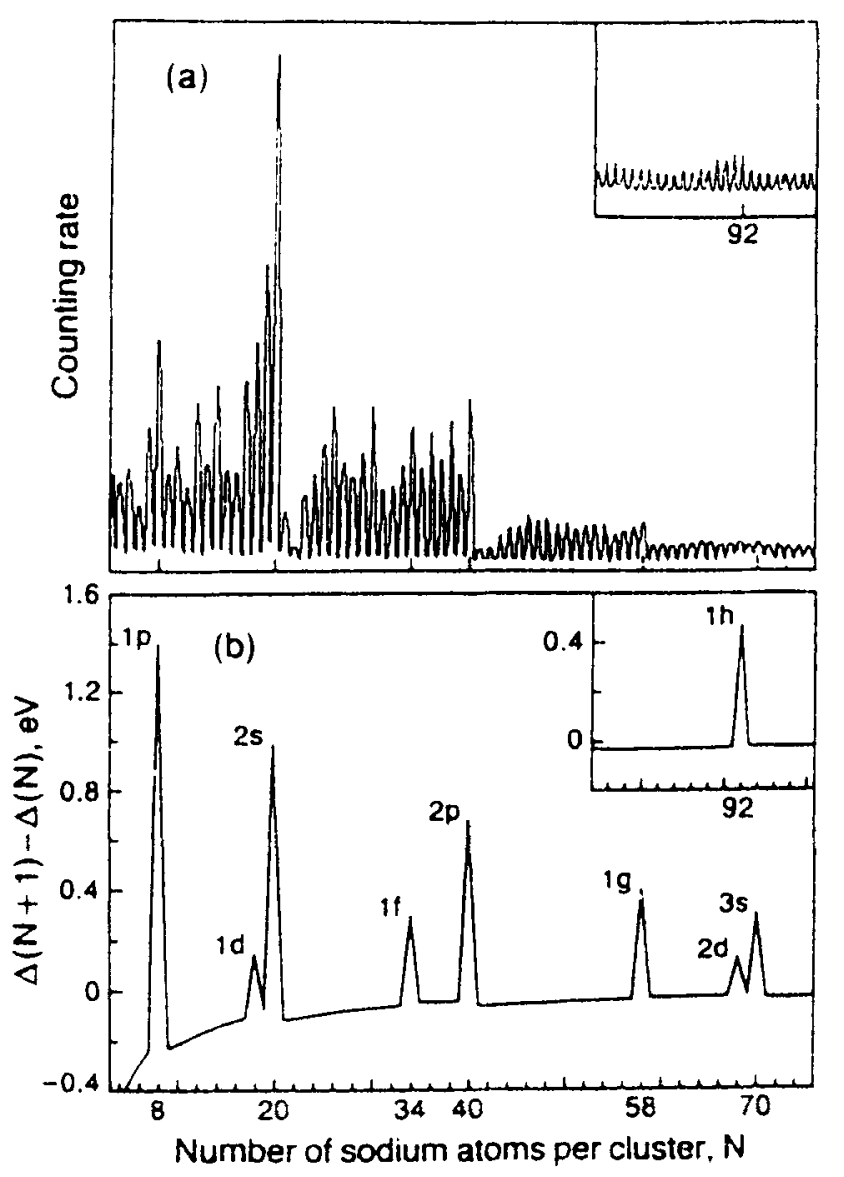

Figure 1. (a) Abundance spectrum of sodium clusters and (b) the difference in energy of $N$ and $N+1$ atom clusters in a spherical potential well. Peaks at $8,20,40$ and 58 correspond to magic clusters (after Knight et al 1984).

and Gong and Kumar (1993) considered substitutional doping of $\mathrm{Al}_{13}$ with tetravalent elements such as $\mathrm{Si}, \mathrm{Ge}$ and $\mathrm{C}$ to make it a 40 valence electron cluster. In figure $3 \mathrm{a}$ we show the binding energies of some 40 valence electron icosahedral $\mathrm{Al}_{12} \mathrm{X}$ clusters (with $\mathrm{X}=\mathrm{Si}, \mathrm{Ge}, \mathrm{C}, \mathrm{Al}, \mathrm{B}, \mathrm{Ga}, \mathrm{As}$, at the centre) as a function of the centre to vertex distance $R$. Figure $3 \mathrm{~b}$ shows the corresponding one electron eigenvalues obtained (Gong and Kumar 1993) by solving the Kohn-Sham equations (Kohn and Sham 1965) within the local density approximation (LDA). It is noted that doping with a tetravalent atom leads to a contraction in the bond length and a large gain in energy which makes these clusters very stable and interesting for considering as superatoms. The electronic structure has features as expected from the spherical jellium model for $\mathrm{Al}_{13}^{-}$. The $1 f$ state splits into a 4-and a 3-fold degenerate states. In a few other cases some states get interchanged. The HOMO-LUMO gap is about $2 \mathrm{eV}$ for all the 40 valence electron clusters. This results in a low reactivity of these clusters similar to the rare gas atoms and it has been confirmed (Leuchtner et al 1989) from studies of oxygen exposure on $\mathrm{Al}_{\mathrm{N}}^{-}$clusters that oxygen reacts with all other aluminum clusters except the magic clusters, $\mathrm{Al}_{13}^{-}$and $\mathrm{Al}_{23}^{-}$. However, since the HOMO-LUMO gap is small as compared 


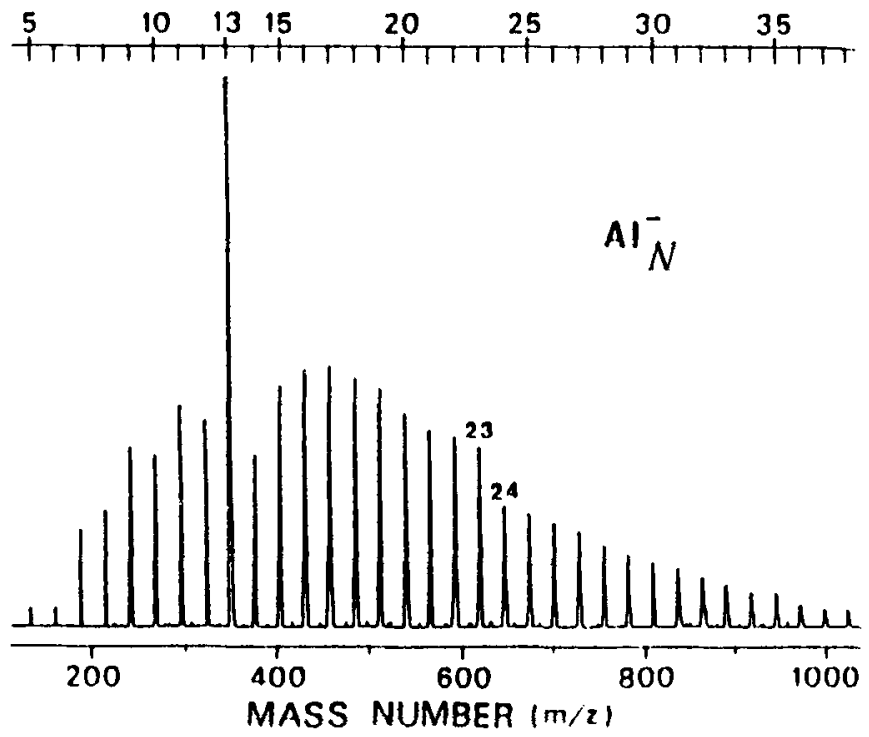

Figure 2. Time of flight mass spectrum of $\mathrm{Al}_{\mathrm{N}}^{-}(\mathrm{N}=5-38)$ clusters. One peak at $\mathrm{N}=13$ and a step between $\mathrm{N}=23$ and 24 corresponding to magic clusters can be seen (After Nakajima et al 1991a).

to the value for rare gases, we expect the properties of solids made of such superatoms to be different from rare gas solids.

\subsection{Fullerenes}

A celebrated example of magic clusters of a non-metallic system is of fullerenes. These are caged clusters of carbon with 12 pentagonal and a varying number of hexagonal rings (Kroto 1987). The smallest member of this family is $\mathrm{C}_{20}$, having a dodecahedron structure. Thereafter the mass spectrum shows abundance of only the clusters with even number of atoms. As shown in figure 4, under certain experimental conditions, it is possible to produce almost only $\mathrm{C}_{60}$. This has 20 hexagonal rings and all the atoms have identical environment with three nearest neighbours forming a very stable nearly spherical truncated icosahedral structure. All the bonds within a pentagon are long (single bonds of length $\approx 1.45 \AA$ ) whereas the remaining bonds are double bonds with approximately 1.40 $\AA$ length (Yannoni et al 1991). Similar to graphite, each atom in $C_{60}$ is covalently bonded with three neighbouring atoms and the $p_{z}$ orbitals give rise to the $\pi$ bonded valence states. The HOMO is five-fold degenerate whereas the LUMO has the $t_{1 u}$ symmetry (Saito and Oshiyama 1991) with a gap of about $1.9 \mathrm{eV}$.

2.2a Doped fullerenes: Several efforts have been made (Heath et al 1985; Guo et al 1991; Smalley 1992) to dope fullerenes with the dopants inside, outside and on the fullerenes. Heath et al (1985) produced some lanthanum complexes and later Chai et al (1991) studied doping of fullerenes with La, K, and B. It was shown from photofragmentation that the metal atoms were encapsulated inside the cage. $\mathrm{La}$ in $\mathrm{C}_{82}$ was found to be uniquely stable. Evidence for coalescence of such fullerenes at high 

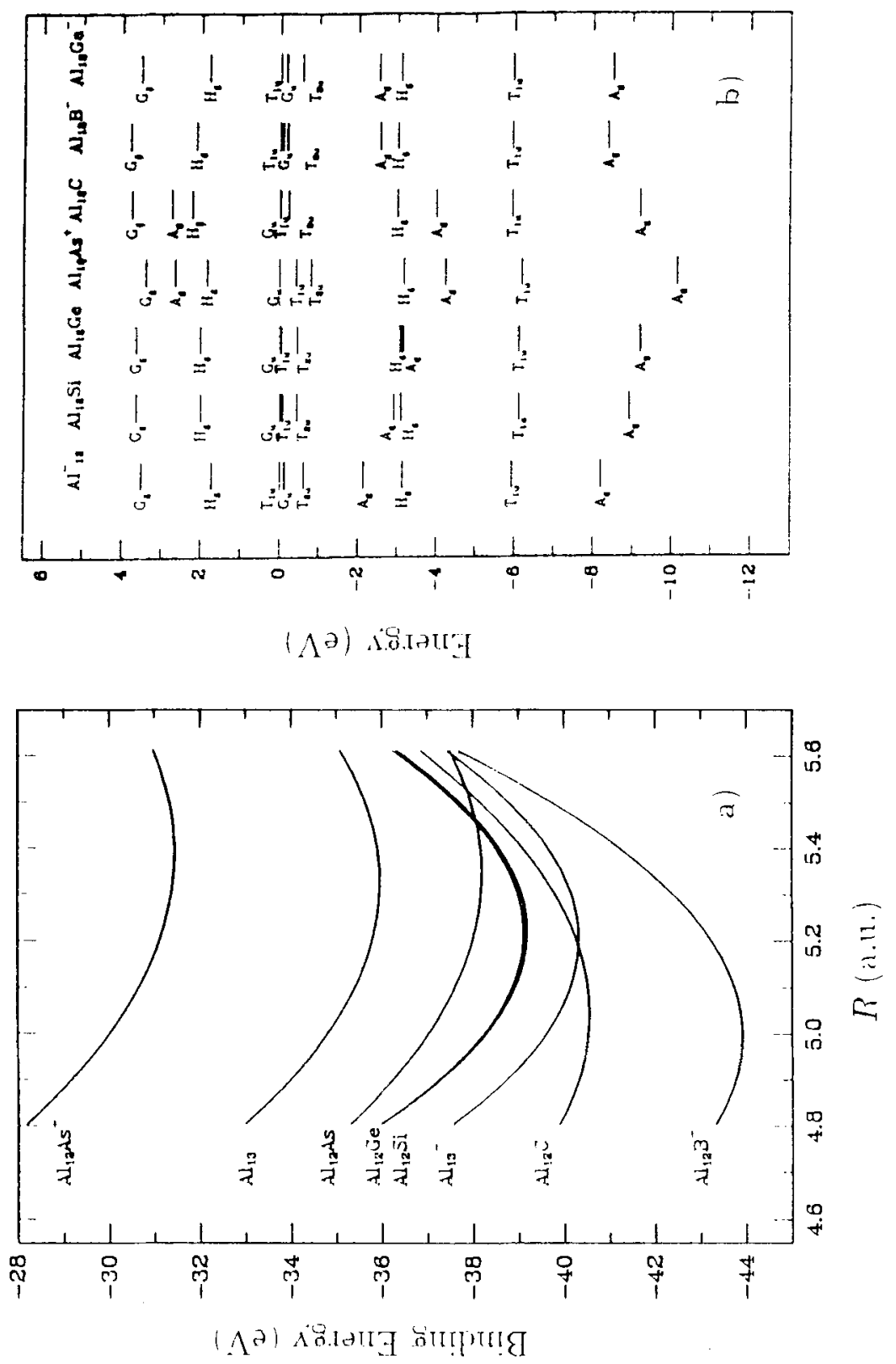

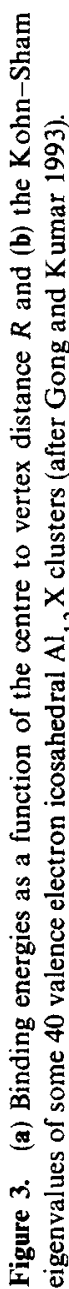



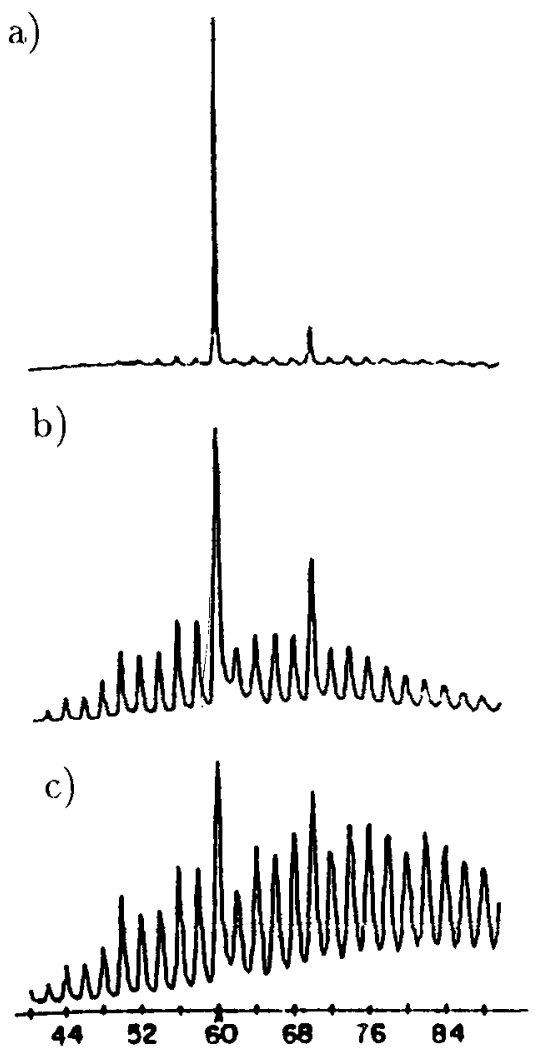

No. of carbon atoms per cluster

Figure 4. Time of flight mass spectra of carbon clusters obtained from laser vaporization of graphite. The three spectra differ in the extent of helium collisions in the supersonic nozzle. In (c), the effective helium density over the graphite target was less than 10 torr while in (b) roughly 760 torr was present. The enhancement of $\mathrm{C}_{60}$ and $\mathrm{C}_{70}$ clusters was believed to be due to gas phase reactions. The spectrum in (a) was obtained by maximizing these cluster thermalization and cluster-cluster reactions. The main feature of this spectrum is the occurrence of $\mathrm{C}_{60}$ as the main specie with a small fraction of $\mathrm{C}_{70}$ (after Kroto et al 1985).

temperatures was also obtained which led to formation of larger fullerenes with as many as three lanthanum atoms inside. In an independent study Yannoni et al (1992) reported encapsulation of scandium clusters in fullerene cages. These endohedral fullerenes, represented (Chai et al 1991; Smalley 1992) by the formula $M @ C_{\mathrm{N}}$ ( $M=$ encapsulated atom), have created much excitement as these could lead to the development of some novel materials but unlike $\mathrm{C}_{60}$, isolation of such fullerenes has been difficult due to their reactive nature.

2.2b Adsorption on fullerenes: Similar to benzene, several derivatives of fullerenes have been developed. Among the simplest, it has been found (see Dunlap et al 1991) that up to 36 atoms of hydrogen could be associated with $\mathrm{C}_{60}$. Oxygen leads to a weakening of the $\mathrm{C}-\mathrm{C}$ bonds. This has been used to insert guest atoms in the $\mathrm{C}_{60}$ cage. Recently Zimmermann et al (1994) observed that 32 atoms of divalent metals such as 
$\mathrm{Ca}, \mathrm{Ba}, \mathrm{Sr}$ on $\mathrm{C}_{60}$ lead to a particularly abundant cluster. In the case of $\mathrm{C}_{70}$, a similar situation occurs with 37 divalent metal atoms. These correspond to the number of pentagonal and hexagonal rings in $\mathrm{C}_{60}$ and $\mathrm{C}_{70}$, and, therefore, $i$ thas been suggested to be a way of finding the number of such rings in a fullerene if one assumes that the metal atoms get adsorbed on the pentagonal and hexagonal sites. Gong and Kumar (1995) recently studied the electronic structure of calcium covered $\mathrm{C}_{60}$ and found that the particular abundance of such clusters is due to geometrical rather than electronic factor. There is a charge transfer of about 12 electrons from $\mathrm{Ca}$ atoms to $\mathrm{C}_{60}$, while the remaining electrons participate in the bonding between metal atoms giving rise to a large density of states at the $E_{\mathrm{F}}$. There is a significant hybridization between the $\mathrm{Ca}$ and $\mathrm{C}_{60}$ states. This mixed ionic-covalent bonding leads to a large binding energy of about $5 \mathrm{eV}$ per $\mathrm{Ca}$ atom. On the other hand for $12 \mathrm{Li}$ atoms adsorbed on $\mathrm{C}_{60}$, the bonding is completely ionic as the 12 electrons get completely transferred to $\mathrm{C}_{60}$ (Kohanoff et al 1992; Gong and Kumar 1995). The charge transfer also makes the two bond lengths in $\mathrm{C}_{60}$ nearly equal but the change is very small. On the other hand $\mathrm{K}$ adsorption on graphite leads to about 0.75 electron transfer from a $\mathrm{K}$ atom at low coverage (Ancilotto and Toigo 1993) and a binding energy of about $0.6 \mathrm{eV}$ only as compared to $2 \cdot 6 \mathrm{eV}$ for $\mathrm{Li}$ adsorption on $\mathrm{C}_{60}$. Though the charge transfer for each carbon atom of $\mathrm{C}_{60}$ and graphite may be similar, the stronger ionic character of fullerene gives rise to a larger binding energy and this should lead to different properties of metal covered fullerenes as compared to metal adsorption on graphite.

The result that the stability of metal covered fullerenes is not due to electronic factors, suggests that $\mathrm{C}_{60}$ can be considered as a curved surface and it should be possible to cover fullerenes even with transition metals or rare earths also to produce some novel clusters with metallic shells which could exhibit magnetic or mixed valence properties very different from their flat surfaces. Also fullerenes could provide a unique way for getting high dispersion of metal atoms for catalytic applications.

\subsection{Metallo-carbohedrenes}

Guo et al $(1992 \mathrm{a}, \mathrm{b})$ studied transition metal doped carbon clusters and found strong abundance of $\mathrm{M}_{8} \mathrm{C}_{12}(\mathrm{M}=\mathrm{V}, \mathrm{Zr}$, Hf, and Ti). These metallo-carbohedrenes, were suggested to have a structure similar to $\mathrm{C}_{20}$ in which each metal atom has three carbon atoms as nearest neighbours, the metal-carbon bond length being different from the carbon-carbon bond length. These studies have opened up new possibilities for making other stable molecules which could possibly be used to form new materials.

\section{Clusters as the basis for developing new materials}

The electronic structure of superatoms can differ very significantly from the one in atoms and bulk as there can be states with 4 -fold and 5-fold symmetry and the separation between the eigenstates and their nature could in general be very different from atoms leading to very different hybridizations in their solids. Also the structure of superatoms could lead to novel forms of materials. Therefore, properties of the solids made from such superatoms could be very different than the ones obtained from atoms as the basis. Also similar to atoms, the states near the highest occupied level would play a dominant role in the bonding whereas the deeper lying states are expected to behave 
like the core states. This behaviour would, however, depend upon the total width of the band and the nature of states which can vary significantly from cluster to cluster. In the following we discuss a few examples.

The special abundance of $\mathrm{C}_{60}$ led to the discovery of the molecular form of carbon, the solid $\mathrm{C}_{60}$ which has the $\mathrm{Pa} \overline{3}$ structure at low temperatures (David et al 1991). It undergoes a transition to an orientationally disordered fcc phase at room temperature. The HOMOs and LUMOs of $\mathrm{C}_{60}$ form narrow bands with a width of about $0.5 \mathrm{eV}$ and a direct band gap of about $1.5 \mathrm{eV}$ (Saito and Oshiyama 1991) in the fcc phase. Bonding between the fullerenes is predominantly of van der Waals nature. The single and the double bonds give rise to small charge transfer from single to double bond regions which lead to a weak electrostatic interaction between the fullerenes resulting in a specific orientational order. Similarly the rugby ball shaped $\mathrm{C}_{70}$ fullerene solidifies with the closed packed hcp and fcc structures.

An important milestone in the research on fullerenes took place when superconductivity was observed at relatively high temperatures (Hebard et al 1991; Rosseinsky et al 1991) with alkali metal doping. In the metallic $A_{3} C_{60}(A=K, R b)$ phase the three electrons of the alkali atoms half-fill the $t_{1 u}$ derived conduction bands. The doping leads to an expansion of the lattice, and, therefore, narrower bandwidth. This increases the density of states at the Fermi level which has been correlated with the increase in the superconducting transition temperature (Schlüter et al 1992).

Similar to carbon fullerenes, there is interest to produce similar molecules of other elements such as $\mathrm{Si}, \mathrm{B}$ and $\mathrm{N}$ or $\mathrm{BH}$ and $\mathrm{BN}$ complexes. In an interesting development Yamanaka et al (1995) synthesized $\mathrm{Na}_{x} \mathrm{Ba}_{y} \mathrm{Si}_{46}$ phase, which can be described as a bcc lattice of dodecahedral $\mathrm{Na} @ \mathrm{Si}_{20}$ clusters. The 6 slightly distorted tetrahedral interstitial sites per $\mathrm{Si}_{20}$ cluster are occupied by $3 \mathrm{Ba}$ and $3 \mathrm{Si}$ atoms such that all the $\mathrm{Si}$ atoms are tetrahedrally coordinated. Subsequently Kawaji et al (1995) observed superconductivity in this Si network material with the transition temperature of $4 \mathrm{~K}$. Saito and Oshiyama (1995) studied the electronic structure of the $\mathrm{Na}_{2} \mathrm{Ba}_{6} \mathrm{Si}_{46}$ phase. As shown in figure $5 \mathrm{a}$, the pristine $\mathrm{Si}_{46}$ solid has an LDA gap of $1.26 \mathrm{eV}$ which is very different from the value of $0.54 \mathrm{eV}$ in the diamond structure of Si. Since LDA underestimates band gap, the correct gap is likely to be about $1.9 \mathrm{eV}$, which is comparable to the value for porous $\mathrm{Si}$ and lies in the visible light range. Also there is a smaller gap within the valence band region, which does not exist in the diamond structure. On the other hand, the cohesive energy of $\mathrm{Si}_{46}$ is only $0.09 \mathrm{eV}$ per atom smaller than in the diamond structure. Doping with $\mathrm{Na}$ and $\mathrm{Ba}$ leads to a charge transfer from metal atoms to $\mathrm{Si}_{20}$
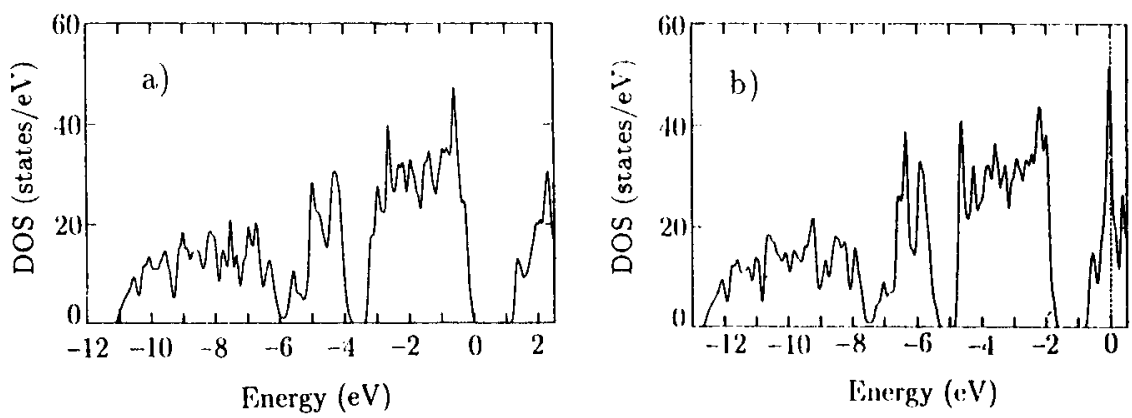

Figure 5. Electronic density of states of (a) the pristine $\mathrm{Si}_{46}$ solid and (b) $\mathrm{Na}_{2} \mathrm{Ba}_{6} \mathrm{Si}_{46}$ (after Saito and Oshiyama 1995). 
and a large density of states at the $E_{\mathrm{F}}$ (figure $5 \mathrm{~b}$ ) due to hybridization of $\mathrm{Ba}$ and $\mathrm{Si}_{20}$ states. It could be responsible for superconductivity in this material. This development has further enhanced the possibilities of synthesizing other fullerene based materials.

Calculations on Mg (Kumar and Car 1991; Khanna and Jena 1992) and Sb (Sundararajan and Kumar 1995) clusters indicate that magic clusters with a complete shell bind weakly with other atoms/clusters, though there can be structural modifications in some cases. $\mathrm{Sb}_{4}$ clusters are interesting as there is a phase in which antimony tetramers are weakly bonded. Whether closed shell magic clusters would form van der Waals bonded solids similar to solid $\mathrm{C}_{60}$ or become metallic as $\mathrm{Mg}$, depends upon the HOMO-LUMO gap and the overlap of the HOMOs and LUMOs. Calculations by Seitsonen et al (1992) on a model fcc $\mathrm{Al}_{12}$ Si solid show that it becomes a metal. If such a phase would exist in reality remains to be seen. Here it should be mentioned that there is a $\mathrm{Al}_{12} \mathrm{~W}$ phase in which icosahedral $\mathrm{Al}_{12} \mathrm{~W}$ clusters are distributed on a bcc lattice (Pearson 1973). This is a unique example of a naturally occurring cluster material and suggests that one should also explore the possibilities of synthesizing cluster materials directly as the corresponding clusters may be difficult to synthesize or isolate. An important question is whether the clusters would coalesce when assembled because such structures are metastable. However, if the clusters are strongly bound then it is likely that the metastable structures could remain in the same structure for long enough period. More work on other systems would be helpful to explore these possibilities.

\section{Bonding in cluster assembled materials}

Besides the van der Waals bonding in solids derived from superatoms as discussed above and the possibilities of a non-metal-metal transition such as in $\mathrm{Mg}$ in going from atoms to solids (Kumar and Car 1991), solids of superatoms differ in an important way from those of atoms because even though bonding between the superatoms could be weak, the nature of bonding within a cluster/molecule could be for e.g. metallic as it is in Al clusters or covalent as in fullerenes and this could lead to very different properties of such materials as compared to materials with atoms as the basis. In cases where the magic clusters do not have a completely filled shell such as $\mathrm{Al}_{12} \mathrm{~B}$, the clusters are expected to be reactive and can accept or give electrons to form cluster compounds. Thus $\mathrm{Al}_{12} \mathrm{~B}$ with a deficiency of one electron should behave like a chlorine atom and interact with a $\mathrm{Na}$ atom as $\mathrm{NaCl}$ so that there is a transfer of an electron from $\mathrm{Na}$ to $\mathrm{Al}_{12}$ B. In a model calculation Khanna and Jena (1994) studied bcc $\mathrm{KAl}_{13}$, having a binding energy of about $5 \mathrm{eV}$ even though there is no miscibility of $\mathrm{K}$ in Al. Unlike the usual ionic solids, the change in the size of the clusters due to charge transfer is small as compared to atoms. In fact calculations on $\mathrm{Al}_{13}^{-}$cluster led to a contraction of bond lengths as compared to expansion normally found in atoms whereas positively charged $\mathrm{Al}_{12} \mathrm{As}^{+}$clusters have a slight expansion (Gong and Kumar 1993). This is due to the redistribution of the charge between the ions in the cluster. In other situations where even after charge transfer the electronic configuration does not correspond to a shell closing, then the remaining electrons could participate in bonding between clusters. This can lead to fusion of clusters as it is known in the case of cluster compounds (Simon 1981).

Saito and Ohnishi (1987) studied binding between two $\mathrm{Na}_{19}$ clusters using a jellium model. As $\mathrm{Na}_{18}$ corresponds to a shell closing, $\mathrm{Na}_{19}$ is expected to behave as 

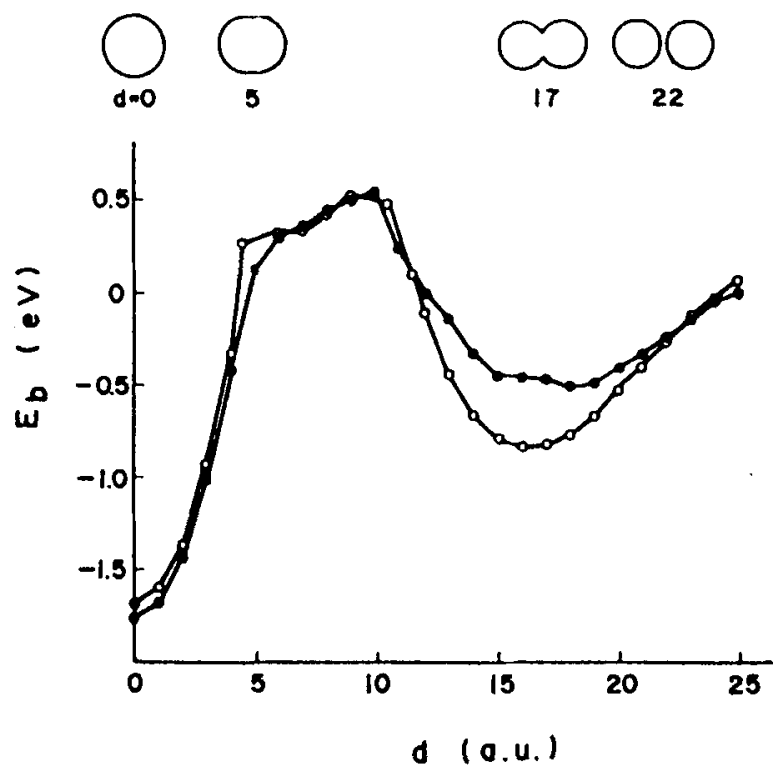

Figure 6. Binding energy curves of $\left(\mathrm{Na}_{19}\right)_{2}$ for two different electronic configurations. In the jellium model the ionic charge in each cluster has been replaced by a spherical positive background. The filled (open) circles correspond to a triplet (singlet) state. Schematic pictures for the positive background are shown for several inter-jellium distances $d$ (after Saito and Ohnishi 1987).

a monovalent atom. Accordingly, their calculations showed (figure 6) a covalent bonding between the two $\mathrm{Na}_{19}$ clusters. However, this is a metastable state as the configuration in which the two clusters coalesce is energetically more favourable. Therefore, the (dynamic) transition barrier for coalescence will play an important role in determining if the clusters could be assembled in metastable structures.

\section{Summary}

In summary, we have presented some recent results where clusters are used as the building blocks of new materials. Besides $\mathrm{C}_{60}$ and some other fullerenes whose solids have already been prepared, clusters in the family of $\mathrm{Al}_{12} \mathrm{Si}$ and metcar have been studied in detail and these hold promise. There are other materials which are also made of clusters such as $\mathrm{Al}_{12} \mathrm{~W}, \mathrm{Na}_{2} \mathrm{Ba}_{6} \mathrm{Si}_{46} \ldots$ and can be viewed as cluster assembled materials. Clusters present in such materials and magic clusters with partially filled shells may be difficult to produce in some cases either due to their reactive nature or excess strain such as in small fullerenes which may get released due to the presence of other atoms leading to a change in bonding nature or hybridization in the solid. Therefore, instead of first producing clusters and then solids, the direct route of producing cluster assembled materials is also promising, and in this regard the work on cluster compounds is very relevant. While we need to do more experimental work on the possibilities of producing other clusters and molecules in abundance, computer simulations can play an important role in finding a basis for developing new 
clusters/molecules. As the atomic and electronic structure of such superatoms is in general different from the bulk, the properties of solids derived from them are likely to be very different from those with atoms as basis. The bonding between clusters shows similar behaviour as between atoms such as van der Waals, ionic, covalent ... but the charge transfer effects are different. The superatoms could lead to novel structures and we hope that further studies in these directions would help in the development of new classes of materials.

\section{Acknowledgements}

I thank X G Gong for several enlightening discussions. I am grateful to the editors for their patience and to Dr Baldev Raj for inviting me to the Indian National Science Congress.

\section{References}

Ancilotto F and Toigo F 1993 Phys. Rev. B47 13713

Bednorz J G and Muller K A 1986 Z. Phys. B-Condensed Matter 64189

Burcham W E 1973 Nuclear physics: An introduction (London: Longman)

Chai Y et al 1991 J. Phys. Chem. 957564

David W I F et al 1991 Nature 353147

de Heer W A, Knight W D, Chou M Y and Cohen M L 1987 Solid State Physics (eds) H Ehrenreich and D Turnbull (New York: Academic Press) 40 p. 93

Dunlap B I, Brenner D W, Mintmire J W, Mowrey R C and White C T 1991 J. Phys. Chem. 955763

Echt O, Sattler K and Recknagel E 1981 Phys. Rev. Lett. 471121

Gong X G and Kumar V 1993 Phys. Rev. Lett. 702078

Gong X G and Kumar V 1994 Phys. Rev. B50 17701 (and to be published)

Gong X G and Kumar V 1995 unpublished

Guo B C, Kerns K P and Castleman Jr. A W 1992a Science 2551411

Guo B C, Wei S, Purnell J, Buzza S and Castleman Jr. A W 1992b Science 256515

Guo T, Jin C and Smalley R E 1991 J. Phys. Chem. 954948

Heath J R, O'Brien S C, Zhang Q, Liu Y, Curl R F. Kroto H W, Tittel F K and Smalley R E 1985 J. Am. Chem. Soc. 1077779

Hebard A F, Rosseinsky M J, Haddon R C, Murphy D W, Glarum S H, Palstra T T M, Ramirez A P and Kortan A R 1991 Nature 350600

Jena P, Khanna S N and Rao B K (eds) 1992 Physics and chemistry of finite systems: from clusters to crystals (Netherlands: Kluwer Academic Publishers) Vol. $1 \& 2$

Jiang L, Li J, Nagahara L A, Kino N, Kitazawa K, Iyoda T, Hashimoto K and Fujishima A 1995 Appl. Phys. A61 17

Kawaji H, Horie H, Yamanaka S and Ishikawa M 1995 Phys. Rev. Lett. 741427

Khanna S N and Jena P 1992 Phys. Rev. Lett. 691664

Khanna S N and Jena P 1994 Chem. Phys. Lett. 219479

Knight W D, Clemenger K, de Heer W A. Sanders W A, Chou M Y and Cohen M L 1984 Phys. Rev. Lett. 52 2141

Kohanoff J, Andreoni W and Parrinello M 1992 Chem. Phys. Lett. 198472

Kohn W and Sham L J 1965 Phys. Rev. A140 1133

Krätschmer W, Lamb L D. Fostiropoulos K and Huffman D R 1990 Nature 347354

Kroto H W 1987 Nature 329529

Kroto H W, Heath J R. O'Brien S C, Curl R F and Smalley R E 1985 Nature 318162

Kumar V 1994 in Lectures on methods of electronic structure calculations (eds) V Kumar, O K Andersen and A Mookerjee (Singapore: World Scientific) p. 317

Kumar V 1995 in Icosahedral symmetry in materials (eds) S Ranganathan. K Chattopadhyay and P Ramachandrarao (Pergamon Press) 
Kumar V and Car R 1991 Phys. Rev. B44 8243

Kumar V, Martin T P and Tosatti E (eds) 1993 Clusters and fullerenes (Singapore: World Scientific)

Leuchtner R E, Harms A C and Castleman Jr. A W 1989 J. Chem. Phys. 912753

Morss L R and Fuger J (eds) 1992 Transuranium elements: a half century (Washington, DC: American Chemical Society)

Nakajima A, Kishi T, Sugioka T, Sone Y and Kaya K 1991a Chem. Phys. Lett. 177297

Nakajima A, Kishi T, Sugioka T and Kaya K 1991b Chem. Phys. Lett. 187239

Pearson W B 1973 The crystal chemistry and physics of metals and alloys (New York: Wiley)

Prasad R, Kumar V and Sundararajan V 1995 (unpublished)

Rosseinsky M J et al 1991 Phys. Rev. Lett. 662830

Saito S and Ohnishi S 1987 Phys. Rev. Lett. 59190

Saito S and Oshiyama A 1991 Phys. Rev. Lett. 662637

Saito S and Oshiyama A 1995 Phys. Rev. B51 2628

Schlüter M, Lannoo M, Needels M, Baraff G A and Tománek D 1992 J. Phys. Chem. Solids 531473

Seaborg G T 1963 Man-made transuranium elements (Englewood Cliffs, NJ: Prentice-Hall, Inc.)

Seitsonen A P, Puska M J, Alatalo M, Nieminen R M, Milman V and Payne M C 1992 Phys. Rev. B48 1981

Shechtman D, Blech I, Gratias D and Cahn J W 1984 Phys. Rev. Lett. 531951

Simon A 1981 Angew. Chem. Int. Ed. Engl. 201

Smalley R E 1992 in ACS Symposium Series, 481 Fullerenes (eds) G S Hammond and V J Kuck (Washington: American Chemical Society) p. 141

Sundararajan V and Kumar V $1995 \mathrm{~J}$. Chem. Phys. (in press)

Wei S, Guo B C, Purnell J, Buzza S and Castleman Jr. A W 1992 Science 256818

Wu M K et al 1987 Phys. Rev. Lett. 58908

Yamanaka S, Horie H, Nakano H and Ishikawa M 1995 Fullerene Sci. Technol. 321

Yannoni C S, Bernier P P, Bethune D S, Meijer G and Salem J R 1991 J. Am. Chem. Soc. 1133190

Yannoni C S, Hoinkis M, de Vries M S, Bethune D S, Salem J R, Crowder M S and Johnson R D 1992 Science 2561191

Yi J Y, Oh D J and Bernholc J 1991 Phys. Rev. Lett. 671594

Zhou O et al 1991 Nature 351462

Zimmermann U, Malinowski N, Näher U, Frank S and Martin T P 1994 Phys. Rev. Lett. 723542

Note added in proof: Chandrabhas et al, Phys. Rev. Lett. 73, 3411 (1994) developed single crystals of $\mathrm{C}_{70}$ and studied phase transitions under pressure using Raman spectroscopy. Beyers et al, Science 370, 196 (1994) reported the preparation of pure crystalline $\mathrm{Sc}_{2} @ \mathrm{C}_{84}$ endohedral metallofullerenes. At room temperature the $\mathrm{Sc}_{2} @ \mathrm{C}_{84}$ molecules pack in a hcp structure with a ratio of lattice constant $c / a=1.63$. Interestingly the molecular spacing of $11.2 \AA$ is the same as in the case of crystalline $C_{84}$. Whetten et al, Adv. Mater. 8, 428 (1996) have recently prepared gold nanocrystals passivated by self-assembled monolayers of straight-chain alkylthiolate molecules in varying size and developed nanocrystal superlattices. The nanocrystals were characterized to have fcc gold lattices and morphology of a dominant truncated-octahedral motif. Arrays of semiconductor nanocrystals are being prepared (Science 271, 920 (1996)) to develop nanostructured lasers. Kumar (unpublished) has studied adsorption of $\mathrm{Li}, \mathrm{Al}, \mathrm{Si}, \mathrm{P}, \mathrm{S}$ and $\mathrm{Cl}$ on $\mathrm{Al}_{7}$ and $\mathrm{Al}_{13}$ clusters. $\mathrm{Al}_{7}$ is expected to behave like an alkali atom whereas $\mathrm{Al}_{13}$, like a halogen. As expected $\mathrm{Li}$ and $\mathrm{Cl}$ have different bonding on the two clusters. 